\title{
Association of albuminuria and reduced estimated glomerular filtration rate with incident stroke and coronary artery disease in patients with type 2 diabetes
}

\author{
Ryotaro Bouchi, Tetsuya Babazono, Naoshi Yoshida, Izumi Nyumura, Kiwako Toya, Toshihide Hayashi, \\ Ko Hanai, Nobue Tanaka, Akiko Ishii and Yasuhiko Iwamoto
}

It is unclear whether albuminuria and reduced glomerular filtration rate (GFR) independently increase the risk of incident stroke and coronary artery disease (CAD) in Japanese patients with diabetes. We investigated the independent effects of albuminuria and estimated GFR (eGFR) on the first occurrence of stroke and CAD in patients with type 2 diabetes mellitus (T2DM). We studied 1002 T2DM patients with eGFR $\left(\mathrm{ml} \mathrm{min}^{-1}\right.$ per $\left.1.73 \mathrm{~m}^{2}\right) \geqslant 15$ and had no previous cardiovascular disease (CVD) history. GFR was estimated using the modified three-variable equation for the Japanese. Patients were divided into four eGFR categories: $\geqslant 90,60-89,30-59$ and $15-29$. The end point was an incident stroke and CAD events. The Cox proportional hazard model was used to calculate hazard ratio and $95 \%$ confidence interval. During a mean follow-up period of $5.2 \pm 2.1$ years, 72 episodes of stroke and 90 of CAD were observed. Multivariate Cox analysis revealed no significant association between the eGFR category and incident stroke. The stroke hazard ratio (95\% confidence interval) in reference to patients with an eGFR $\geqslant 90$ was 0.78 (0.40-1.56) for patients with an eGFR of 60-89, $1.47(0.70-3.10)$ for patients with an eGFR of 30-59 and 1.14 (0.39-3.35) for patients with an eGFR of 15-29. Reduced eGFR was a significant risk factor for CAD, with hazard ratios (95\% confidence interval) for patients with an eGFRs of 60-89, 30-59 and 15-29 at 1.81 (1.01-3.57), 2.03 (1.04-4.40) and 3.01 (1.13-8.02), respectively. Reduced eGFR is independently associated with incident CAD but not stroke in Japanese patients with T2DM.

Hypertension Research (2010) 33, 1298-1304; doi:10.1038/hr.2010.170; published online 30 September 2010

Keywords: albuminuria; coronary artery disease; diabetes; estimated glomerular filtration rate; stroke

\section{INTRODUCTION}

Chronic kidney disease $(\mathrm{CKD})^{1}$ has been recognized as an independent risk factor for cardiovascular disease (CVD) mortality and morbidity both in the Japanese ${ }^{2}$ and Western populations. ${ }^{3,4}$ It has also been demonstrated that decreased estimated glomerular filtration rate (eGFR) is an independent risk factor for cardiovascular events and all-cause-mortality in patients with type 2 diabetes mellitus (T2DM) ${ }^{5}$ Observational analyses from the Action in Diabetes and Vascular Disease: preterAx and diamicroN-MR Controlled Evaluation study ${ }^{6}$ indicated that albuminuria and reduced eGFR, important components of CKD, are separately and independently associated with incident CVD. Similar findings were observed in patients with T2DM; however, there are no available data regarding whether albuminuria and reduced GFR are, independently of each other, associated with incident CVD in Japanese patients with T2DM.

A post hoc analysis from the Prevention of Renal and Vascular End-Stage Disease study ${ }^{7}$ showed that a category of subjects with a decreased eGFR but normoalbuminuria was not at increased risk of CVD, and the impact of reduced GFR was shown by PROSPER (Prospective study of Pravastatin in the Elderly at Risk) to be greater for coronary artery disease (CAD) than for stroke in elderly people. ${ }^{8}$ We have recently shown that albuminuria, but not reduced eGFR, is associated with not only incident symptomatic stroke ${ }^{9}$ but also prevalent silent cerebral infarction ${ }^{10}$ in patients with T2DM. These findings ${ }^{8-10}$ may indicate the different impact of reduced GFR on incident stroke and CAD. In the present study, we aimed to examine the independent association of albuminuria and reduced eGFR, with incident stroke and CAD in Japanese patients with T2DM.

\section{METHODS}

Study population

This prospective hospital-based observational cohort study was conducted in accordance with the Declaration of Helsinki. The study comprised consecutive patients with T2DM who were admitted to the Department of Medicine, 
Diabetes Center, Tokyo Women's Medical University Hospital in Tokyo, Japan between 1 January 2002 and 31 December 2003. Patients were 20 years of age or older, and were admitted for glycemic control and for the evaluation of diabetic complications. Patients undergoing renal replacement therapy, pregnant women, and patients with infectious and malignant diseases were excluded. T2DM was diagnosed according to criteria from the World Health Organization. ${ }^{11}$ We have previously studied 1300 type 2 diabetic patients who had no history of symptomatic stroke and the eGFR was $\geqslant 15 \mathrm{ml} \mathrm{min}^{-1}$ per $1.73 \mathrm{~m}^{2}$, regardless of the degree of urinary albumin excretion in the same period to evaluate the association between CKD components and incident stroke. ${ }^{9}$ From the same cohort, we selected patients who had no history of symptomatic stroke, CAD or peripheral artery disease in the present study.

Participants underwent a routine medical history, physical examination and blood sampling. Information regarding smoking and family history of CVD was obtained using a standard questionnaire. Smoking habits were classified as current or not. Physical examination included blood pressure measurement and anthropometry; laboratory examinations included hemoglobin $\mathrm{AlC}$, serum lipids and creatinine using fasting blood samples; and urinary albumin was measured in the first morning urine specimen.

Past history of CVD was defined as stroke/transient ischemic attack, CAD and peripheral artery disease. Past history of stroke and transient ischemic attack was obtained from medical records. Clinical evidence of CAD was defined from the following: angina pectoris diagnosed by coronary angiography or myocardial scintigraphy and a history of myocardial infarction or previous coronary revascularization. Past history of peripheral artery disease was defined as lower extremity peripheral artery disease according to American College of Cardiology/American Heart Association 2005 guidelines. $^{12}$

\section{Measurements}

Serum creatinine was initially measured by Jaffe's method in the hospital laboratory. From January 2003, this method was replaced by an enzymatic method, and measurements were adjusted using a regression equation obtained from the correlational analysis between both measurements of serum creatinine analysis in 10132 samples from diabetic patients: serum creatinine (enzymatic method, $\mathrm{mg} \mathrm{dl}^{-1}$ ) $=0.972 \times$ serum creatinine (Jaffe's method, $\mathrm{mg} \mathrm{dl}^{-1}$ ) -0.224 $(r=0.9992, P<0.001)$.

GFR was estimated using the following modified three-variable equation for the Japanese, as has recently been proposed by the Japanese Society of Nephrology: ${ }^{13} \mathrm{GFR}=194 \times \mathrm{SCr}^{-1.094} \times \mathrm{age}^{-0.287}$ ((if female) $\left.\times 0.739\right)$, where $\mathrm{SCr}=$ serum creatinine in $\mathrm{mg} \mathrm{dl}^{-1}$, measured by an enzymatic method. Patients were divided into four eGFR categories: eGFR $\geqslant 90,60-89,30-59$ and $15-29 \mathrm{ml} \mathrm{min}^{-1}$ per $1.73 \mathrm{~m}^{2}$. Classification of the degree of urinary albumin was assessed according to the American Diabetes Association criteria, on the basis of the albumin-to-creatinine ratio (ACR) in the first morning urine specimen. ${ }^{14}$ Urinary ACR was calculated from urinary albumin, as determined using the latex agglutination method, and urinary creatinine concentrations. Normoalbuminuria, microalbuminuria and macroalbuminuria were defined as an ACR $<30,30-299$ and $\geqslant 300 \mathrm{mg} \mathrm{g}^{-1}$, respectively.

Hemoglobin A1C was determined using the high-performance liquid chromatography (normal range: $4.3-5.8 \%$ ). Total cholesterol and high-density lipoprotein cholesterol were determined enzymatically. Low-density lipoprotein cholesterol was calculated using the Friedewald's equation, when serum triglycerides level was $<4.57 \mathrm{mmoll}^{-1}$. $^{15}$

\section{Outcomes}

Patients were followed up until September 2009. The primary end point was an incident first stroke and CAD event, information on which was obtained by direct reference to medical records by a single investigator (RB). Stroke was defined as an acute focal neurological deficit lasting longer than $24 \mathrm{~h}$ or resulting in death within $24 \mathrm{~h}$ of the onset of symptoms, and was diagnosed as being due to cerebral lesions of vascular origin (International Classification of Diseases, 9th Revision (ICD-9), codes of cerebrovascular diseases). Most stroke cases were diagnosed by computed tomography, MRI including diffusion image, magnetic resonance angiography of the brain and carotid duplex imaging. CAD was defined as myocardial infarction, angina pectoris of which patients received treatment in hospital for ischemic discomfort and diagnosed by coronary angiography, coronary revascularization and sudden death. Coronary revascularization was achieved when a patient underwent percutaneous coronary intervention (for example, angioplasty, stenting, atherectomy and laser ablation) or coronary artery bypass graft.

\section{Statistical analyses}

Data were expressed as percentage, arithmetic mean \pm s.d. or geometric mean with $95 \%$ confidence interval, as appropriate, according to data distribution. Triglycerides and ACR were logarithmically transformed owing to skewed distributions. For statistical analyses, one-way analysis of variance was used for between-group comparisons for continuous variables and the CochranArmitage test for categorical variables. The cumulative incidence of the end point was estimated using the Kaplan-Meier method. Risk estimates for reaching the end point were calculated using univariate and multivariate Cox proportional hazard model analyses. The following variables were incorporated as covariates: age, sex, duration of diabetes, presence of proliferative diabetic retinopathy, smoking status, body mass index, systolic and diastolic blood pressures, usage of renin-angiotensin system inhibitors (angiotensin-converting enzyme inhibitors and angiotensin receptor blockers), anti-platelet agents and statins, hemoglobin $\mathrm{A} 1 \mathrm{C}$, triglycerides, high-density lipoprotein cholesterol, low-density lipoprotein cholesterol, hemoglobin, uric acid, eGFR and urinary ACR at baseline. All statistical analyses were performed using the Statistical Analysis System (SAS Institute, Cary, NC, USA) version 9.13. A $P$-value $<0.05$ was considered significant.

\section{RESULTS}

A total of 1002 T2DM patients had sufficient baseline and follow-up data to qualify for inclusion in the study, including 452 women and 550 men with a mean ( \pm s.d.) age of $61 \pm 12$ years (range: $21-90$ years). At baseline, the mean eGFR was $76.6 \pm 26.6 \mathrm{ml} \mathrm{min}^{-1}$ per $1.73 \mathrm{~m}^{2}$ (range: 16.6-216.1), and 283, 469, 203 and 47 patients were classified into eGFR groups $\geqslant 90,60-89,30-59$ and $15-29 \mathrm{ml} \mathrm{min}^{-1}$ per $1.73 \mathrm{~m}^{2}$, respectively. Demographic and characteristic patient data in four eGFR categories are listed in Table 1.

Compared with patients with an eGFR $\geqslant 90 \mathrm{ml} \mathrm{min}^{-1}$ per $1.73 \mathrm{~m}^{2}$, those with lower eGFRs were likely to be older, have exhibited a longer duration of diabetes and have higher systolic but lower diastolic blood pressure, yielding a higher pulse pressure. Furthermore, reduced eGFR was significantly associated with a higher prevalence of diabetic retinopathy. Medications and laboratory data according to eGFR levels are shown in Tables 1 and 2, respectively.

During the mean follow-up period of $5.2 \pm 2.1$ years $($ median $=6.1$ years; maximum $=7.8$ years; overall $=5247$ patient-years), 72 first episodes of stroke were observed, yielding an incidence rate of 13.7 episodes per 1000 patient-years. The stroke cases were classified as 65 with cerebral infarction, 6 with cerebral bleeding and 1 with subarachnoid hemorrhage. In all, 90 first episodes of CAD (17.2 episodes per 1000 patient-years), including 12 myocardial infarctions, 63 angina pectoris and 15 sudden deaths, were also observed. Of 51 coronary interventions, 40 cases were percutaneous coronary intervention and 11 were coronary artery bypass graft. As shown in Figure 1a, the 5-year cumulative incidence of stroke in patients with an eGFR $\geqslant 90,60-89,30-59$ and $15-29$ were $3.9,5.4,13.6$ and $18.3 \%$, respectively $(P<0.001$ by the log-rank test). Those of CAD were 3.6, $6.1,12.0$ and $27.4 \%$, respectively (Figure $1 \mathrm{~b} ; P<0.0001$ by the logrank test). When incident stroke and $\mathrm{CAD}$ events were combined as a composite end point (total CVD), the 5-year cumulative incidence of total CVD in patients with an eGFR $\geqslant 90,60-89,30-59$ and 15-29 were $7.2,10.6,20.8$ and $41.6 \%$, respectively (Figure $1 c ; P<0.0001$ by the log-rank test).

Table 3 shows the estimated hazard ratios of stroke, CAD and total CVD relative to the category of an eGFR $\geqslant 90 \mathrm{ml} \mathrm{min}^{-1}$ per $1.73 \mathrm{~m}^{2}$ for other categories of eGFR in patients with T2DM based on 
Table 1 Clinical characteristics and concomitant medication in type 2 diabetic patients according to eGFR levels

\begin{tabular}{|c|c|c|c|c|c|}
\hline \multirow[b]{2}{*}{ Characteristic } & \multicolumn{5}{|c|}{ eGFR $\left(\mathrm{m} / \mathrm{min}^{-1}\right.$ per $\left.1.73 \mathrm{~m}^{2}\right)$} \\
\hline & $\geqslant 90(n=283)$ & $60-89(n=469)$ & $30-59(n=203)$ & $15-29(n=47)$ & P-value \\
\hline Sex (male, \%) & 56.5 & 54.2 & 55.2 & 51.1 & 0.595 \\
\hline Family history of CVD (\%) & 27.6 & 37.3 & 29.6 & 25.5 & 0.904 \\
\hline Current smoker (\%) & 29.7 & 23.7 & 19.7 & 21.3 & 0.014 \\
\hline Systolic blood pressure $(\mathrm{mm} \mathrm{Hg})$ & $128 \pm 11$ & $130 \pm 19$ & $135 \pm 23$ & $147 \pm 28$ & $<0.001$ \\
\hline Diastolic blood pressure $(\mathrm{mm} \mathrm{Hg})$ & $77 \pm 11$ & $75 \pm 12$ & $73 \pm 13$ & $79 \pm 16$ & 0.007 \\
\hline Pulse pressure $(\mathrm{mm} \mathrm{Hg})$ & $51 \pm 16$ & $54 \pm 17$ & $62 \pm 19$ & $68 \pm 23$ & $<0.001$ \\
\hline Proliferative diabetic retinopathy (\%) & 22.6 & 30.9 & 43.8 & 63.8 & $<0.001$ \\
\hline Atrial fibrillation (\%) & 3.2 & 5.5 & 4.9 & 4.3 & 0.429 \\
\hline \multicolumn{6}{|l|}{ Anti-hypertensive agents (\%) } \\
\hline Calcium channel blockers & 19.1 & 25.4 & 38.4 & 76.6 & $<0.001$ \\
\hline ACEIs & 13.8 & 18.6 & 25.2 & 21.3 & 0.004 \\
\hline ARBs & 13.8 & 22.4 & 37.4 & 51.2 & $<0.001$ \\
\hline$\beta$-Blockers & 3.5 & 3.2 & 7.4 & 8.5 & 0.020 \\
\hline$\alpha$-Blockers & 0.7 & 3.2 & 2.5 & 12.8 & 0.001 \\
\hline Diuretics & 1.1 & 5.2 & 21.2 & 38.3 & $<0.001$ \\
\hline Anti-platelet agents (\%) & 13.4 & 22.6 & 32.0 & 23.4 & $<0.001$ \\
\hline Statins $(\%)$ & 20.9 & 24.7 & 34.0 & 23.4 & 0.012 \\
\hline
\end{tabular}

Abbreviations: ACEI, angiotensin-converting enzyme inhibitor; ARB, angiotensin type 1-receptor blocker; CVD, cardiovascular disease.

Data are expressed as mean \pm s.d. or as percentage.

${ }^{a}$ ANOVA or Cochran-Armitage test.

Table 2 Laboratory data in type 2 diabetic patients according to eGFR levels

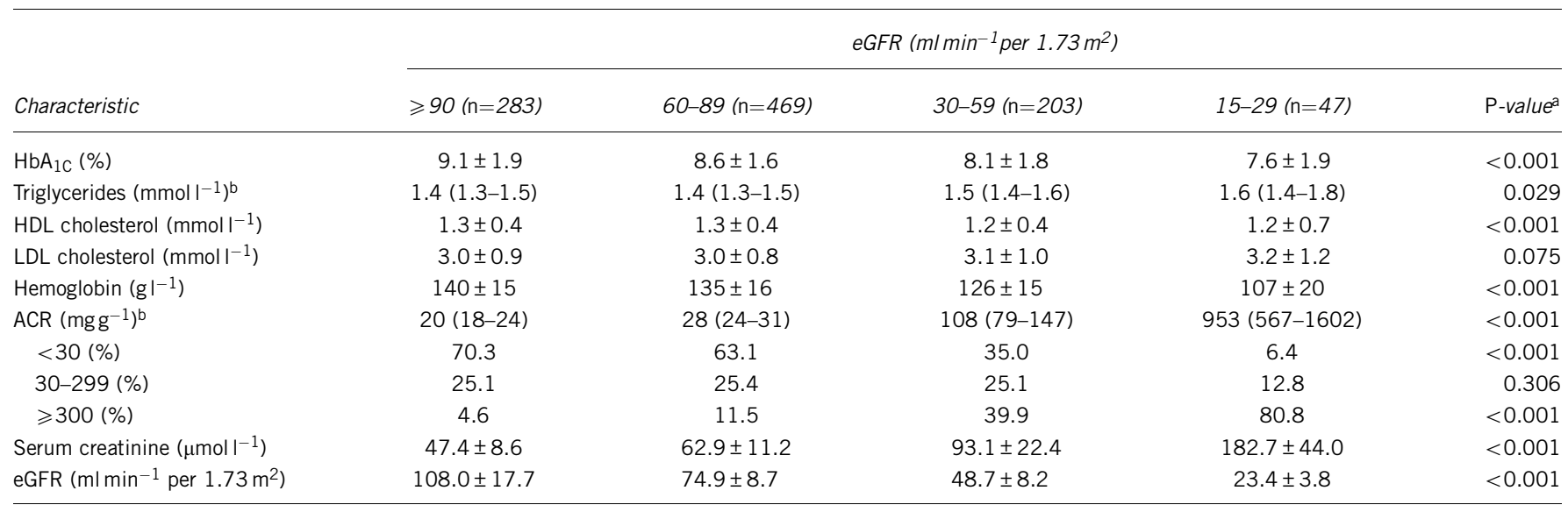

Abbreviations: ACR, albumin-to-creatinine ratio; eGFR, estimated glomerular filtration rate; HDL/LDL, high/low density-lipoprotein.

Data are expressed as mean \pm s.d., geometric mean $(95 \% \mathrm{Cl})$ or percentage.

aANOVA or Cochran-Armitage test.

${ }^{\mathrm{b}} \mathrm{Geometric} \mathrm{mean.}$

univariate and multivariate Cox regression analyses. Stroke hazard ratios for patients with an eGFR of 30-59 and $15-29 \mathrm{ml} \mathrm{min}^{-1}$ per $1.73 \mathrm{~m}^{2}$ were significant in the univariate Cox model; however, no statistical significance was evident from multivariate Cox analysis of hazard ratios for patients with an eGFR of 30-59 and 15-29 $\mathrm{ml} \mathrm{min}^{-1}$ per $1.73 \mathrm{~m}^{2}$. In contrast, reduced eGFR was a significant risk factor for $\mathrm{CAD}$ in both the univariate and multivariate Cox analyses including albuminuria as a covariate. When data were analyzed combining stroke and CAD events as total CVD events, there were also significant increases in total CVD risk in the eGFR of 30-59 and $15-29 \mathrm{ml} \mathrm{min}^{-1}$ per $1.73 \mathrm{~m}^{2}$ groups in multivariate Cox regression analysis. 
a

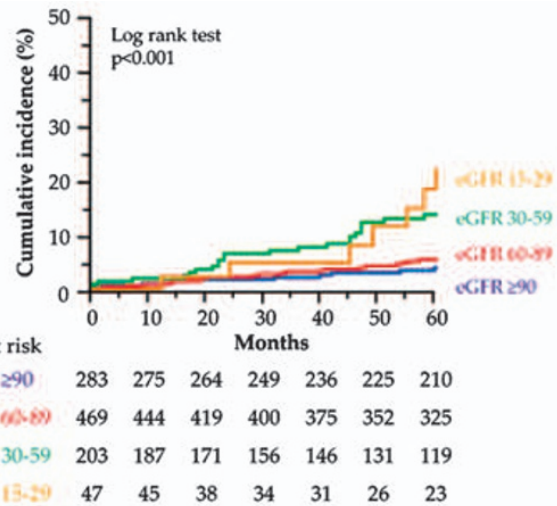

b

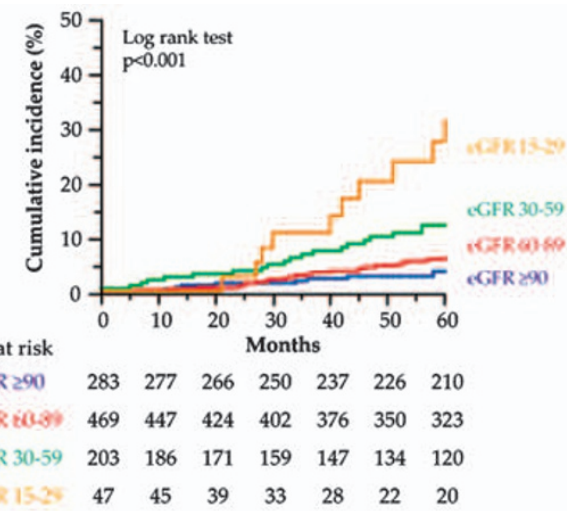

C

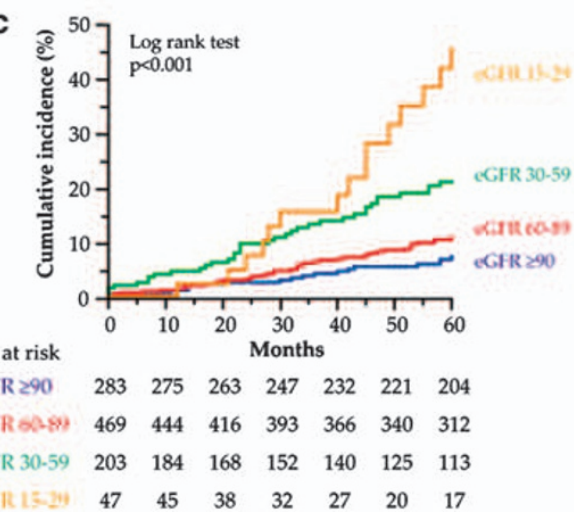

Figure 1 Kaplan-Meier curve of cumulative incidence of first stroke (a), CAD (b) and total CVD including stroke and CAD (c) events in patients with type 2 diabetes stratified by eGFR: eGFR $\geqslant 90,60-89,30-59$ and $15-29 \mathrm{ml} \mathrm{min}^{-1}$ per $1.73 \mathrm{~m}^{2}$.

Table 3 Hazard ratios of stroke, coronary artery disease and total cardiovascular disease in patients with type 2 diabetes stratified by eGFR categories

\begin{tabular}{|c|c|c|c|c|c|c|c|c|c|}
\hline \multirow[b]{2}{*}{ eGFR $\left(\mathrm{m} / \mathrm{min}^{-1}\right.$ per $\left.1.73 \mathrm{~m}^{2}\right)$} & \multicolumn{3}{|c|}{ Stroke } & \multicolumn{3}{|c|}{$C A D$} & \multicolumn{3}{|c|}{ Total CVD } \\
\hline & $H R$ & $95 \% \mathrm{Cl}$ & $\mathrm{P}$-value & $H R$ & $95 \% \mathrm{Cl}$ & P-value & $H R$ & $95 \% \mathrm{Cl}$ & P-value \\
\hline$\geqslant 90$ & 1.00 & (Reference) & & 1.00 & (Reference) & & 1.00 & (Reference) & \\
\hline $60-89$ & 1.21 & $0.62-2.37$ & 0.621 & 2.38 & $1.22-4.63$ & 0.011 & 1.66 & $1.02-2.68$ & 0.040 \\
\hline $30-59$ & 3.36 & $1.73-6.51$ & $<0.001$ & 4.35 & $2.16-8.74$ & $<0.001$ & 3.61 & $2.20-5.93$ & $<0.001$ \\
\hline \multicolumn{10}{|l|}{ Multivariate analysis } \\
\hline$\geqslant 90$ & 1.00 & (Reference) & & 1.00 & (Reference) & & 1.00 & (Reference) & \\
\hline $60-89$ & 0.79 & $0.40-1.56$ & 0.494 & 1.81 & $1.01-3.57$ & 0.049 & 1.23 & $0.75-2.02$ & 0.407 \\
\hline $30-59$ & 1.47 & $0.70-3.10$ & 0.312 & 2.03 & $1.04-4.40$ & 0.042 & 1.66 & $1.01-2.89$ & 0.045 \\
\hline $15-29$ & 1.14 & $0.39-3.35$ & 0.810 & 3.01 & $1.13-8.02$ & 0.027 & 1.86 & $1.07-3.97$ & 0.040 \\
\hline $\log A C R\left(\mathrm{mgg}^{-1}\right)$ & 1.85 & $1.36-2.53$ & $<0.001$ & 1.48 & $1.13-1.93$ & 0.004 & 1.75 & $1.39-2.22$ & $<0.001$ \\
\hline Gender (male vs. female) & & NS & & & NS & & 1.56 & $1.10-2.22$ & 0.013 \\
\hline Body mass index $\left(\mathrm{kg} \mathrm{m}^{-2}\right)$ & & NS & & & NS & & 1.04 & $1.00-1.09$ & 0.046 \\
\hline
\end{tabular}

Abbreviations: ACR, albumin-to-creatinine ratio; CAD, coronary artery disease; Cl, confidence interval; CVD, cardiovascular disease; eGFR, estimated glomerular filtration rate; HR, hazard ratio; LDL, low density-lipoprotein. 


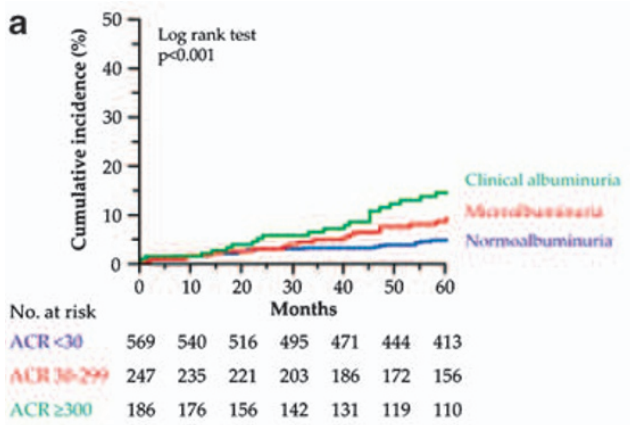

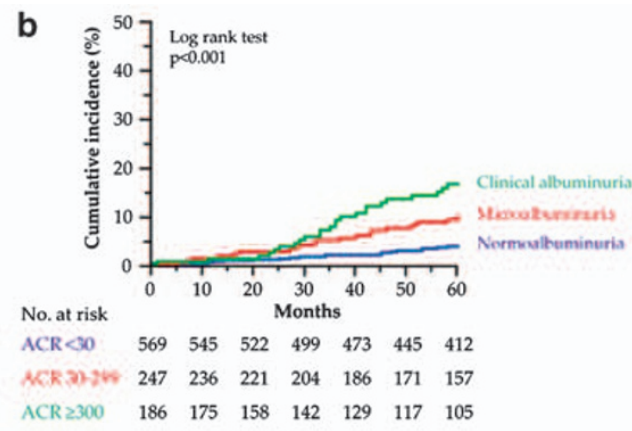

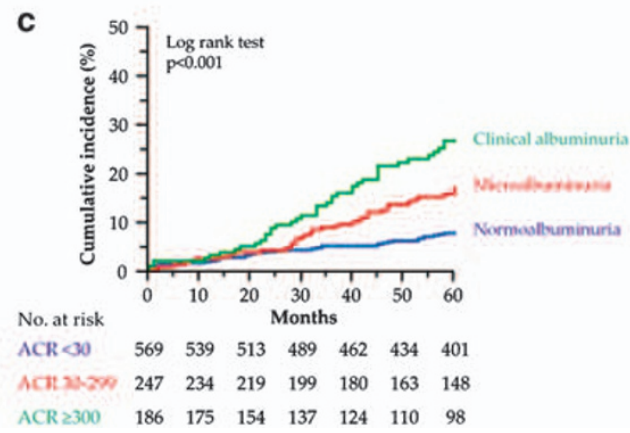

Figure 2 Kaplan-Meier curve of cumulative incidence of first stroke (a), CAD (b) and total CVD including stroke and CAD (c) events in patients with type 2 diabetes stratified by urinary ACR: ACR $<30 \mathrm{mgg}^{-1}$, normoalbuminuria; $30-299 \mathrm{mgg}^{-1}$, microalbuminuria; $\geqslant 300 \mathrm{mgg}^{-1}$, clinical albuminuria.

Table 4 Hazard ratios of stroke, coronary artery disease and total cardiovascular disease in patients with type 2 diabetes stratified by urinary ACR levels

\begin{tabular}{|c|c|c|c|c|c|c|c|c|c|}
\hline \multirow[b]{2}{*}{ ACR $\left(m g g^{-1}\right)$} & \multicolumn{3}{|c|}{ Stroke } & \multicolumn{3}{|c|}{$C A D$} & \multicolumn{3}{|c|}{ Total CVD } \\
\hline & $H R$ & $95 \% \mathrm{Cl}$ & P-value & $H R$ & $95 \% \mathrm{Cl}$ & P-value & $H R$ & $95 \% \mathrm{Cl}$ & P-value \\
\hline \multicolumn{10}{|l|}{ Univariate analysis } \\
\hline$<30$ & 1.00 & (Reference) & & 1.00 & (Reference) & & 1.00 & (Reference) & \\
\hline 30-299 & 2.06 & $1.56-3.69$ & 0.014 & 2.56 & $1.34-3.80$ & 0.002 & 2.15 & $1.46-3.27$ & $<0.001$ \\
\hline$\geqslant 300$ & 3.59 & $2.07-6.22$ & $<0.001$ & 3.93 & $2.39-6.44$ & $<0.001$ & 3.67 & $2.50-5.41$ & $<0.001$ \\
\hline$<30$ & 1.00 & (Reference) & & 1.00 & (Reference) & & 1.00 & (Reference) & \\
\hline 30-299 & 2.02 & $1.11-3.65$ & 0.021 & 1.97 & $1.16-3.34$ & 0.012 & 2.33 & $1.52-3.57$ & $<0.001$ \\
\hline$\geqslant 300$ & 4.60 & $2.31-9.13$ & $<0.001$ & 2.70 & $1.46-4.99$ & 0.002 & 3.70 & $2.21-6.23$ & $<0.001$ \\
\hline eGFR $\left(\mathrm{ml} \mathrm{min}^{-1}\right.$ per $\left.1.73 \mathrm{~m}^{2}\right)$ & 1.00 & $0.99-1.01$ & 0.541 & 0.99 & $0.98-1.00$ & 0.008 & 0.99 & $0.98-1.00$ & 0.013 \\
\hline Age (years) & 1.06 & $1.04-1.09$ & $<0.001$ & 1.03 & $1.01-1.05$ & 0.003 & 1.06 & $1.04-1.07$ & $<0.001$ \\
\hline Family history of CVD & 1.69 & $1.05-2.71$ & 0.030 & & NS & & & NS & \\
\hline Atrial fibrillation & & NS & & & NS & & 1.81 & $1.01-3.24$ & 0.047 \\
\hline RAS inhibitors & & NS & & & NS & & 0.69 & $0.48-0.99$ & 0.047 \\
\hline
\end{tabular}

Abbreviations: ACR, albumin-to-creatinine ratio; CAD, coronary artery disease; $\mathrm{Cl}$, confidence interval; CVD, cardiovascular disease; eGFR, estimated glomerular filtration rate; HR, hazard ratio; LDL, low density-lipoprotein; RAS, rennin-angiotensin system.

When participants were classified by urinary ACR at baseline, the 5 -year cumulative incidences of stroke in patients with normoalbuminuria, microalbuminuria and clinical albuminuria were 4.3, 8.8 and $14.0 \%$, respectively (Figure $2 \mathrm{a} ; P<0.001$ by the log-rank test). As well as stroke, the five-year cumulative incidence of $\mathrm{CAD}$ (Figure 2b; $P<0.001$ by the log-rank test) and total CVD (Figure 2c; $P<0.001$ by the log-rank test) significantly increased with an increase in urinary
ACR in patients with T2DM. Table 4 shows the hazard ratios of stroke, CAD and total CVD in patients with T2DM stratified by urinary ACR categories in univariate and multivariate Cox regression analyses. Compared with patients with normoalbuminuria $(n=569)$, the hazard ratios of stroke, CAD and total CVD events for those with microalbuminuria $(n=247)$ and clinical albuminuria $(n=186)$ were significant in both univariate and multivariate Cox model analyses. 
a

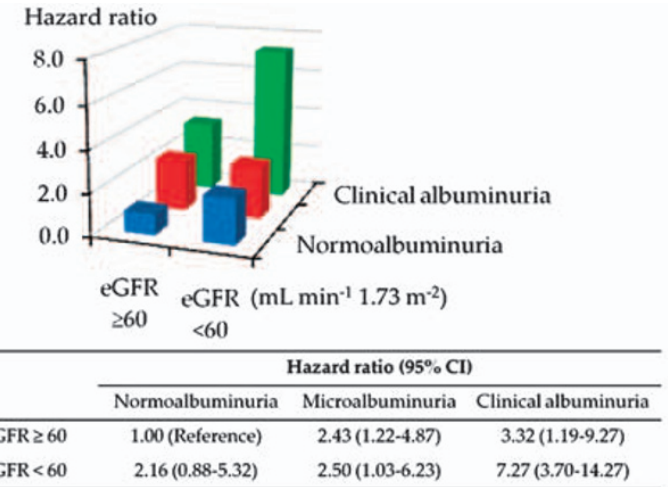

b

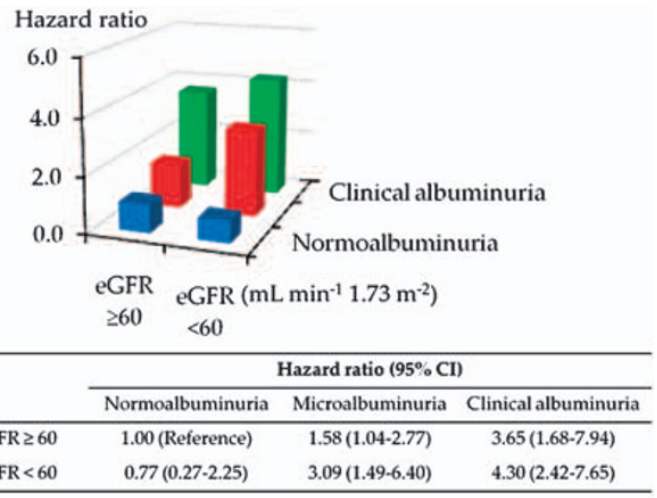

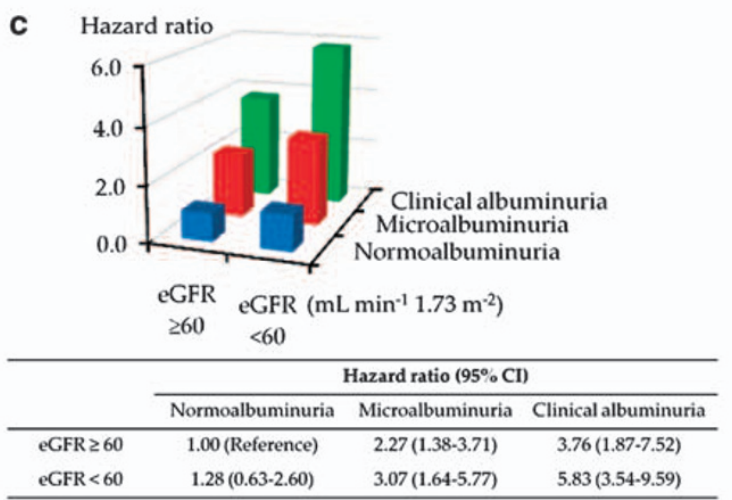

Figure 3 Hazard ratio of stroke (a), CAD (b) and total CVD (c) in patients with type 2 diabetes in the multivariate Cox model in six subgroups stratified by eGFR ( $\geqslant 60 \mathrm{ml} \mathrm{min}^{-1}$ per $1.73 \mathrm{~m}^{2}$ or less) and degree of albuminuria. The multivariate model included covariates listed in the Methods section other than urinary ACR and eGFR.

Finally, hazard ratios were calculated in six subgroups classified according to eGFR ( $\geqslant 60 \mathrm{ml} \mathrm{min}^{-1}$ per $1.73 \mathrm{~m}^{2}$ or less) and category of albuminuria to determine the simultaneous effect of these CKD manifestations on the risk of stroke and CAD events (the subgroup with an eGFR $\geqslant 60 \mathrm{ml} \mathrm{min}^{-1}$ per $1.73 \mathrm{~m}^{2}$ and normoalbuminuria was used as the reference group). As shown in Figure 3a, reduced eGFR was not predictive of incident stroke events except for patients with clinical albuminuria, whereas a stepwise increase in hazard ratios was identified as albuminuria increase, regardless of eGFR levels. In contrast, the impact of reduced eGFR on CAD was observed in patients with microalbuminuria and clinical albuminuria (Figure 3b). As well as stroke, a stepwise increase in hazard ratios was identified as albuminuria increase independent of eGFR levels. Similar findings were observed for total CVD events (Figure 3c). Compared with the reference group, patients with clinical albuminuria and an eGFR $<60 \mathrm{ml} \mathrm{min}^{-1}$ per $1.73 \mathrm{~m}^{2}$ were at the highest risk for each cardiovascular event, whereas those with normoalbuminuria and an eGFR $<60 \mathrm{ml} \mathrm{min}^{-1}$ per $1.73 \mathrm{~m}^{2}$ were not at significantly increased risk for stroke, CAD or total CVD events (Figure 3).

\section{DISCUSSION}

Although CKD has been recently identified as a potent risk factor for cardiovascular events, ${ }^{3,5,16}$ independent effects of the manifestations of CKD, albuminuria and reduced GFR are largely unknown, particularly in diabetic patients who carry a high risk of developing stroke and CAD. To the best of our knowledge, this is the first study to determine the independent effect of albuminuria and eGFR on the incidence of CVD, mainly focusing on stroke and CAD events in patients with T2DM. In this hospital-based prospective cohort study of Japanese diabetic patients, we have demonstrated that reduced eGFR is see- mingly associated with a higher risk of incident stroke and CAD in patients with T2DM. This association remained statistically significant for $\mathrm{CAD}$ and total $\mathrm{CVD}$ even after adjusting for cardiovascular risk factors and urinary ACR; however, the association between reduced eGFR and stroke disappeared. In contrast, albuminuria, another renal manifestation of diabetic kidney disease, was robustly predictive of both stroke and CAD events even after adjusting for covariates including eGFR.

Previous studies ${ }^{5,6}$ have indicated the significant association of reduced eGFR with incident CVD in patients with T2DM even if albuminuria was included as a covariate. Our present findings are compatible with these observations. ${ }^{5,6}$ Reduced GFR has numerous effects on the cardiovascular system, including inhibition of erythropoiesis and platelet function, ${ }^{17,18}$ and induction of volume overload, ${ }^{19}$ dyslipidemia, hypertension ${ }^{20}$ and vascular calcification. ${ }^{21}$ Therefore, reduced GFR is not only a marker of the presence of conventional cardiovascular risk factors but may also have an important role in the pathogenesis of CVD, particularly CAD.

On the other hand, although patients with reduced eGFR had a higher incidence of stroke in the present study, reduced eGFR was not associated with incident stroke events in T2DM patients except those with clinical albuminuria. The higher incidence of stroke in patients with reduced eGFR may be partly due to confounding factors including higher age and diastolic blood pressure (Table 3). In fact, the statistical significance between reduced eGFR and incident stroke disappeared after adjustment for these covariables including albuminuria. Stroke events were less common than coronary events, possibly because of lower associated power to detect a significant association; however, our data were in agreement with those of a pooled analysis of community-based studies. ${ }^{16}$ Bos et al. ${ }^{22}$ from the Rotterdam Study 
also reported that low GFR was not associated with incident ischemic stroke. Furthermore, secondary analysis from PROSPER $^{8}$ indicated that elderly people with reduced GFR were at significantly increased risk of incident CAD but not stroke. These findings suggest that reduced eGFR has less predictive value of incident stroke than CAD. Further studies are required to clarify the different pathogenesis of stroke and CAD associated with reduced eGFR and/or albuminuria.

In contrast to reduced eGFR, albuminuria was confirmed to be a strong and independent predictor of CAD as well as stroke in the present study. This association has been well recognized in previous studies. $^{23-26}$ As discussed previously, albuminuria and CVD share numerous risk factors that may explain this relationship. ${ }^{9}$

The limitations of this study first include an ethnically and socially homogeneous population because of a hospital-based study; therefore, the generalization of our findings may be limited. Second, we only studied diabetic patients without a previous history of CVD to determine the effects of renal parameters in T2DM patients on the first event of stroke or CAD. Our results should therefore be reviewed with caution when extrapolating incidences of secondary stroke or CAD events. Third, we defined patients without CVD by a routine medical history, and procedures such as cranial MRI or myocardial scintigraphy were not performed in all patients in the present study. Therefore, we were unable to rule out the possibility that some of type 2 diabetic patients in the present study may have a history of CVD. Fourth, albuminuria was determined from a single measurement of urinary ACR, possibly leading to improper categorization because of marked day-to-day variability in albumin excretion. Although we did not obtain multiple measurements of urinary ACR, we restricted the timing of urine collection to the first morning urine to minimize exercise-induced and diurnal variation. ${ }^{27}$

\section{CONCLUSION}

This prospective hospital-based observational cohort study has demonstrated that albuminuria and reduced eGFR are independent of each other, associated with CAD but not stroke in Japanese patients with T2DM.

\section{CONFLICT OF INTEREST}

The authors declare no conflict of interest.

1 National Kidney Foundation. K/DOQI clinical practice guidelines for chronic kidney disease: evaluation, classification, and stratification. Kidney Disease Outcome Quality Initiative. Am J Kidney Dis 2002; 39(Suppl. 2): S1-S246.

2 Ninomiya T, Kiyohara Y, Kubo M, Tanizaki Y, Doi Y, Okubo K, Wakagawa Y, Hata J, Oishi Y, Shikata K, Yonemoto K, Hirakata H, lida M. Chronic kidney disease and cardiovascular disease in a general Japanese population: the Hisayama Study. Kidney Int 2005; 68: 228-236.

3 Go AS, Chertow GM, Fan D, McCulloch CE, Hsu CY. Chronic kidney disease and the risks of death, cardiovascular events, and hospitalization. N Eng J Med 2004; 351: 1296-1305.

4 Keith DS, Nichols GA, Vullion CM, Brown JB, Smith DH. Longitudinal follow-up and outcomes among general population with chronic kidney disease in a large managed care organization. Arch Intern Med 2004; 164: 659-663.

5 So WY, Kong AP, Ma RC, Ozaki R, Szeto CC, Chan NN, Ng V, Ho CS, Lam CW, Chow CC, Cockram CS, Chan JC, Tong PC. Glomerular filtration rate, cardiorenal end points, and all-cause-mortality in type 2 diabetic patients. Diabetes Care 2006; 29: 2046-2052.
6 Ninomiya T, Perkovic V, de Galan BE, Zoungas S, Pillai A, Jardine M, Patel A, Cass A, Neal B, Poulter N, Mogensen CE, Cooper M, Marre M, Williams B, Hamet P, Mancia G, Woodward M, Macmahon S, Chalmers J, ADVANCE Collaborative Group. Albuminuria and kidney function independently predict cardiovascular and renal outcomes in diabetes. J Am Soc Nephrol 2009; 20: 1813-1821.

7 Brantsma AH, Bakker SJ, Hillege HL, de Zeeuw D, de Jong PE, Gansevoort RT, PREVEND Study Group. Cardiovascular and renal outcome in subjects with K/DOQI stage 1-3 chronic kidney disease: the importance of urinary albumin excretion. Nephrol Dial Transplant 2008; 23: 3851-3858.

8 Ford I, Bezlyak V, Stott DJ, Sattar N, Packard CJ, Perry I, Buckley BM, Jukema JW, de Craen AJ, Westendorp RG, Shepherd J. Reduced glomerular filtration rate and its association with clinical outcome in older patients at risk of vascular events: secondary analysis. PLoS Med 2009; 6: 76-82.

9 Bouchi R, Babazono T, Nyumura I, Toya K, Hayashi T, Ohta M, Hanai K, Kiuchi Y, Suzuki K, Iwamoto $\mathrm{Y}$. Is a reduced estimated glomerular filtration rate a risk factor for stroke in patients with type 2 diabetes? Hypertens Res 2009; 32: 381-386.

10 Bouchi R, Babazono T, Yoshida N, Nyumura I, Toya K, Hayashi T, Ohta M, Hanai K, Tanaka N, Ishii A, Iwamoto Y. Relationship between chronic kidney disease and silent cerebral infarction in patients with type 2 diabetes. Diabetic Med 2010; 27: 538-543.

11 Alberti KGMM, Zimmet P, for the WHO consultation. Definition, diagnosis, and classification of diabetes mellitus and its complications, part 2: diagnosis and classification of diabetes mellitus provisional report of a WHO consultation. Diabet Med 1996; 15: 539-553.

12 Hirsch AT, Haskal ZJ, Hertzer NR, Bakal CW, Creager MA, Halperin JL, Hiratzka LF, Murphy WRC, Olin JW, Puschett JB, Rosenfield KA, Sacks D, Stanley JC, Taylor Jr LM, White CJ, White J, White RA. ACC/AHA 2005 Practice Guidelines for the Management of Patients With Peripheral Arterial Disease (Lower Extremity, Renal, Mesenteric, and Abdominal Aortic). Circulation 2006; 113: e436-e465.

13 Matsuo S, Imai E, Horio M, Yasuda Y, Tomita K, Nitta K, Yamagata K, Tomino Y, Yokoyama $\mathrm{H}$, Hishida A. Collaborators developing the Japanese equation for estimated GFR. Revised equations for estimated GFR from serum creatinine in Japan. Am J Kidney Dis 2009; 53: 982-992.

14 American Diabetes Association. Nephropathy in diabetes. Diabetes Care 2004; 322 : 531-536.

15 Friedewald WT, Levy RI, Fredrickson DS. Estimation of the concentration of low-density lipoprotein cholesterol in plasma, without use of the preparative ultracentrifuge. Clin Chem 1972; 18: 499-502.

16 Weiner DE, Tighiouart H, Amin MG, Stark PC, MacLeod B, Griffith JL, Salem DN, Levey AS, Sarnak MJ. Chronic kidney disease as a risk factor for cardiovascular disease and all-cause mortality: a pooled-analysis of community-based studies. J Am Soc Nephrol 2004; 15: 1307-1315.

17 Mezzano D, Tagle R, Panes O, Perez M, Downey P, Munoz B, Aranda E, Barja P, Thambo S, Gonzalez F, Mezzano S, Pereira J. Hemostatic disorder of uremia: the platelet defect, main determinant of the prolonged bleeding time, is correlated with indices of activation of coagulation and fibrinolysis. Thromb Haemost 1996; 76: 312-321.

18 Noris M, Remuzzi G. Uremic bleeding: closing the circle after 30 years of controversies? Blood 1999; 94: 2569-2574.

19 Guerin AP, Adda H, London GM, Marchais SJ. Cardiovascular disease in renal failure. Minerva Urol Nefrol 2004; 56: 279-288.

20 Toto RD. Treatment of hypertension in chronic kidney disease. Semin Nephrol 2005; 25: 435-439.

21 Goodman WG, Goldin J, Kuizon BD, Yoon C, Gales B, Sider D, Wang Y, Chung J, Emerick A, Greaser L, Elashoff RM, Salusky IB. Coronary-artery calcification in young adults with end-stage renal disease who are undergoing dialysis. N Eng/ J Med 2000; 342: 1478-1483.

22 Bos MJ, Koudstaal PJ, Hofman A, Breteler MM. Decreased glomerular filtration rate is a risk factor for hemorrhagic stroke but not for ischemic stroke: the Rotterdam Study. Stroke 2007; 38: 3127-3132.

23 Anavekar NS, Gans DJ, Berl T, Rohde RD, Cooper W, Bhaumik A, Hunsicker LG, Rouleau JL, Lewis JB, Vanhille P, Locatelli F, Goldhaber S, Lewis EJ, Pfeffer MA. Predictors of cardiovascular events in patients with type 2 diabetic nephropathy and hypertension: a case for albuminuria. Kidney Int 2004; 92: S50-S55.

24 Dinneen SF, Gerstein HC. The association of microalbuminuria and mortality in noninsulin-dependent diabetes mellitus: a systematic overview of the literature. Arch Intern Med 1997; 157: 1413-1418.

25 Neamer NB, Coull BM, Clark WM, Wynn M. Microalbuminuria in ischemic stroke. Arch Neurol 1999; 56: 699-702.

26 Yuyun MF, Khaw KT, Luben R, Welch A, Bingham S, Day NE, Wareham NJ. Microalbuminuria and stroke in a British population: the European Prospective Investigation into Cancer in Norfolk (EPIC-Norfolk) population study. J Intern Med 2004; 255: 247-255.

27 Babazono T, Takahashi C, Iwamoto Y. Definition of microalbuminuria in first-morning and random spot urine in diabetic patients. Diabetes Care 2004; 27: 1838-1839. 